\title{
Investigation of Effects of Parameters in Element-Free Galerkin Method for Bending Analysis of Mindlin-Reissner Plates
}

\author{
Ö.Y. BozkurT*, M.O.E. Barbar And Ö. ÖZBeK \\ Gaziantep University, Mechanical Engineering Department, Gaziantep, Turkey
}

\begin{abstract}
The element-free Galerkin method is one of the most widely used meshfree method in solid mechanics due to its simplicity and high convergence rate. However, it has some selectable parameters that affect the accuracy and convergence. The parameters can be listed as size of support domain, number of monomials, type of weight function, number of integration points in a background cell and value of penalty coefficient. The effects of these parameters on the accuracy of the element-free Galerkin method solution of the Reissner-Mindlin plate bending are investigated. A number of case studies with regular and irregular node distributions are solved. The displacement and moment values at critical points are compared with exact solutions. It is shown that the selectable parameters have to be carefully selected especially for the analysis of moments.
\end{abstract}

DOI: 10.12693/APhysPolA.128.B-208

PACS: 45.10.-b

\section{Introduction}

The element-free Galerkin (EFG) method, which was developed by Belytschko and co-workers [1], is a popular meshfree method in solid mechanics. The EFG method employs moving least-square (MLS) approximation to construct shape functions. It does not require any element connectivity and the results are not significantly affected by the use of irregular node arrangements. Since MLS shape functions do not possess Kroneckerdelta property, EFG method needs some special techniques such as penalty coefficient [2], Lagrange multipliers [1] for the application of boundary conditions.

In this work, the effects of selectable parameters on the solution accuracy of EFG method for bending analysis of the Reissner-Mindlin plates are investigated. The investigation is carried out using different values of selectable parameters in the solution and comparison of obtained results with results of analytical solutions.

\section{Element-free Galerkin method}

\subsection{Moving least square approximation (MLSA)}

The MLS approximation for the function of a field variable $u(\boldsymbol{x})$ in a local domain $\Omega$ is defined at a point $\boldsymbol{x}$ as

$$
u^{h}(\boldsymbol{x})=\sum_{i=1}^{m} p_{i}(\boldsymbol{x}) a_{i}(\boldsymbol{x})=\boldsymbol{p}^{\mathrm{T}}(\boldsymbol{x}) \boldsymbol{a}(\boldsymbol{x}),
$$

where $\boldsymbol{p}^{\mathrm{T}}(\boldsymbol{x})$ is the vector of monomial basis functions, $a^{\mathrm{T}}(\boldsymbol{x})$ is the vector of coefficients, $\boldsymbol{x}$ is the position vector, and $m$ is the number of basis terms. The difference between the function $u(\boldsymbol{x})$ and its local approximation $u^{h}(\boldsymbol{x})$ must be minimized by weighted discrete $L_{2}$ norm to obtain the vector of coefficients $\boldsymbol{a}(\boldsymbol{x})$ :

*corresponding author; e-mail: oybozkurt@gantep.edu.tr

$$
J=\sum_{i=1}^{n} w\left(\boldsymbol{x}-\boldsymbol{x}_{i}\right)\left[\boldsymbol{p}^{\mathrm{T}}\left(x_{i}\right) \boldsymbol{a}(\boldsymbol{x})-u_{i}\right]^{2},
$$

where $n$ is the number of nodes in the support domain of point $\boldsymbol{x}, u_{i}$ is the nodal value of $u$ at $\boldsymbol{x}=x_{i}, w\left(\boldsymbol{x}-x_{i}\right)$ is the weight function associated with the influence domain of node $i$. The cubic spline and quartic spline weight functions [2] are used in this work. The minimization of weighted residual with respect to $\boldsymbol{a}(\boldsymbol{x})$ at any arbitrary point $\boldsymbol{x}$ can be written as a set of linear equations.

$$
\boldsymbol{A}(\boldsymbol{x}) \boldsymbol{a}(\boldsymbol{x})=\boldsymbol{B}(\boldsymbol{x}) \boldsymbol{U}_{s},
$$

where

$$
\begin{aligned}
& \boldsymbol{A}(\boldsymbol{x})=\sum_{i=1}^{n} w_{i}(\boldsymbol{x}) p\left(x_{i}\right) p^{\mathrm{T}}\left(x_{i}\right), \\
& w_{i}(\boldsymbol{x})=w\left(\boldsymbol{x}-x_{i}\right), \\
& \boldsymbol{B}(\boldsymbol{x})=\left[w_{1}(\boldsymbol{x}) p\left(x_{1}\right),\right. \\
& \left.w_{2}(\boldsymbol{x}) p\left(x_{2}\right), \cdots w_{n}(\boldsymbol{x}) p\left(x_{n}\right)\right], \\
& \boldsymbol{U}_{s}=\left\{u_{1}, u_{2}, u_{3}, \cdots, u_{n}\right\}^{\mathrm{T}} .
\end{aligned}
$$

If the matrix $\boldsymbol{A}$ is non-singular $\boldsymbol{a}(\boldsymbol{x})$ can be written as

$$
\boldsymbol{a}(\boldsymbol{x})=\boldsymbol{A}^{-1}(\boldsymbol{x}) \boldsymbol{B}(\boldsymbol{x}) \boldsymbol{U}_{s} .
$$

The local approximation $u^{h}(\boldsymbol{x})$ can be rewritten by substituting Eq. (7) into Eq. (1):

$$
u^{h}(\boldsymbol{x})=\sum_{i=1}^{n} \phi_{i}(\boldsymbol{x}) u_{i}=\boldsymbol{\Phi}^{\mathrm{T}}(\boldsymbol{x}) \boldsymbol{U}_{\mathrm{s}},
$$

where $\boldsymbol{\Phi}^{\mathrm{T}}$ is the vector of MLS shape functions and it can be expressed as

$$
\begin{gathered}
\boldsymbol{\Phi}^{\mathrm{T}}(x)=\left\{\phi_{1}(x) \phi_{2}(x) \cdots \phi_{n}(x)\right\}= \\
\boldsymbol{p}^{\mathrm{T}}(x) \boldsymbol{A}^{-1}(x) \boldsymbol{B}(x) . \\
\text { 2.2. Governing equations }
\end{gathered}
$$

The discrete system equation for the EFG method solution of the Reissner-Mindlin plate can be written as

$$
\left(\boldsymbol{K}+\boldsymbol{K}^{\alpha}\right) \boldsymbol{U}=\left(\boldsymbol{F}+\boldsymbol{F}^{\alpha}\right),
$$

where $\boldsymbol{K}$ is the global stiffness matrix and is obtained by the assembly of point stiffness matrices 


$$
K_{i j}=\int_{\Omega} \boldsymbol{B}_{i}^{\mathrm{T}} \boldsymbol{D} \boldsymbol{B}_{j} \mathrm{~d} \Omega,
$$

in which

$$
\boldsymbol{B}_{i}=\left[\begin{array}{ccccc}
0 & 0 & 0 & \phi_{i, x} & \phi_{i, y} \\
\phi_{i, x} & 0 & \phi_{i, y} & \phi_{i} & 0 \\
0 & \phi_{i, y} & \phi_{i, x} & 0 & \phi_{i}
\end{array}\right]^{\mathrm{T}}
$$

and $D$ is the elastic material matrix. The $\boldsymbol{K}^{\alpha}$ is the matrix of penalty factors defined by:

$$
\left(\boldsymbol{K}^{\alpha}\right)_{i j}=\int_{\Gamma_{u}} \varphi_{i}^{\mathrm{T}} \boldsymbol{\alpha} \varphi_{j} \mathrm{~d} \Gamma
$$

where $\varphi_{i}$ is a diagonal matrix. If the relevant DOF is free, the diagonal elements of $\varphi_{i}$ are equal to 0, otherwise equal to 1 . The force vector $\boldsymbol{F}$ in Eq. (12) is the global force vector assembled using the nodal force vector of

$$
F_{i}=\int_{\Omega}\left(\boldsymbol{L}_{u} \psi_{i}\right)^{\mathrm{T}} b \mathrm{~d} \Omega+\int_{\Omega}\left(\boldsymbol{L}_{u} \psi_{i}\right)^{\mathrm{T}} t_{\Gamma} \mathrm{d} S,
$$

where $\psi_{i}$ is a diagonal matrix of shape functions. The $\boldsymbol{F}^{\alpha}$ vector shows the forces obtained by the implementation of essential boundary conditions and can be obtained as

$$
F_{i}^{\alpha}=\int_{\Gamma_{u}} \varphi_{i}^{\mathrm{T}} \alpha u_{\Gamma} \mathrm{d} \Gamma
$$

\section{Numerical experiments and discussions}

To investigate the effects selectable parameters of EFG method for the Reissner-Mindlin plate bending problems, two numerical cases are examined: clamped circular plate under uniform transverse distributed load and simply supported square plate under uniform transverse distributed load and are shown in Fig. 1.
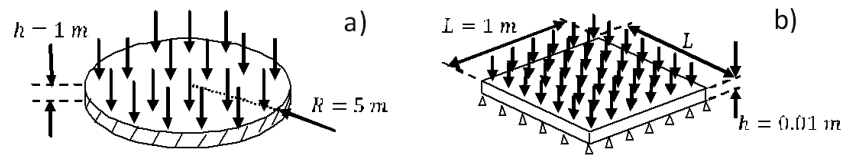

Fig. 1. (a) Clamped circular plate, (b) simply supported square plate.

The material properties and pressure values of the cases are the Young modulus, $E=10920 \mathrm{~Pa}$, the Poisson ratio, $v=0.3$ and $P=1 \mathrm{~Pa}$. The EFG method solutions are compared with analytical solutions.

The regular node distribution with 817 field nodes and 768 background cells were used. Table I and Table II present the normalized deflection results using cubic spline weight function. In the tables, mBasis and $n$ Gauss refer to number of monomial basis and number of Gauss points in a background cell, respectively.

The irregular node distribution with 1089 field nodes and 1024 background cells were used. Table III and Table IV present the normalized moment results using quar-

\begin{tabular}{|c|c|c|c|c|c|c|c|}
\hline \multirow{2}{*}{ mBasis } & \multirow{2}{*}{ nGauss } & \multicolumn{5}{|c|}{ Dimensionless size of support domain $\left(\alpha_{\mathrm{s}}\right)$} & \multirow{2}{*}{ Exact } \\
\hline & & 2.5 & 3.0 & 3.5 & 4.0 & 4.5 & \\
\hline \multirow[t]{5}{*}{3} & $4 \times 4$ & 0.184962 & 0.185032 & 0.185283 & 0.185462 & 0.185478 & $0.184821[3]$ \\
\hline & $5 \times 5$ & 0.184963 & 0.185033 & 0.185283 & 0.185453 & 0.185486 & \\
\hline & $6 \times 6$ & 0.184963 & 0.185033 & 0.185288 & 0.185460 & 0.185478 & \\
\hline & $7 \times 7$ & 0.184963 & 0.185033 & 0.185281 & 0.185460 & 0.185483 & \\
\hline & $8 \times 8$ & 0.184962 & 0.185032 & 0.185285 & 0.185459 & 0.185524 & \\
\hline
\end{tabular}
tic spline weight function.

TABLE I

Central deflections $w_{\mathrm{c}} /\left(p L^{4} / 100 D\right)$ using regular node distribution for clamped circular plate subjected to uniform transverse distributed load.

TABLE II

Central deflections $w_{\mathrm{c}} /\left(p L^{4} / 100 D\right)$ using regular node distribution for clamped circular plate subjected to uniform transverse distributed load. $\alpha_{\mathrm{p}}-$ penalty coefficient $=6$.

\begin{tabular}{c|c|c|c|c|c|c}
\hline \hline \multirow{2}{*}{ mBasis } & \multicolumn{5}{|c|}{ Dimensionless size of support domain $\left(\alpha_{\mathrm{s}}\right)$} & \multirow{2}{*}{ Exact } \\
\cline { 2 - 6 } & 2.5 & 3.0 & 3.5 & 4.0 & 4.5 & \\
\hline 3 & 0.185249 & 0.185321 & 0.185573 & 0.185747 & 0.185768 & 0.184821 \\
6 & 0.185256 & 0.185264 & 0.185378 & 0.185772 & 0.185841 & \\
10 & 0.012204 & 0.016623 & 0.185365 & 0.185837 & 0.186014 &
\end{tabular}


TABLE III

Central moments $M_{\mathrm{c}} /\left(p L^{2} / 10\right)$ using irregular node distribution for simply supported square plate subjected to uniform transverse distributed load.

\begin{tabular}{c|c|c|c|c|c|c|c}
\hline \hline \multirow{2}{*}{ mBasis } & \multirow{2}{*}{ nGauss } & \multicolumn{5}{|c|}{ Dimensionless size of support domain $\left(\alpha_{\mathrm{s}}\right)$} & \multirow{2}{*}{ Exact } \\
\cline { 3 - 7 } & & 2.5 & 3.0 & 3.5 & 4.0 & 4.5 & \\
\hline 3 & $4 \times 4$ & 0.500941 & 0.510744 & 0.519388 & 0.633194 & 0.772233 & $0.4789[3]$ \\
& $5 \times 5$ & 0.487774 & 0.498832 & 0.510473 & 0.497286 & 0.535530 & \\
& $6 \times 6$ & 0.474766 & 0.478865 & 0.479829 & 0.489514 & 0.502671 & \\
& $7 \times 7$ & 0.476700 & 0.476526 & 0.479432 & 0.478829 & 0.480024 & \\
& $8 \times 8$ & 0.477039 & 0.477445 & 0.479317 & 0.479033 & 0.479634 &
\end{tabular}

TABLE IV

Central moments $M_{\mathrm{c}} /\left(p L^{2} / 10\right)$ using irregular node distribution for simply supported square plate subjected to uniform transverse distributed load. $\alpha_{\mathrm{p}}$ - penalty coefficient $=6$.

\begin{tabular}{c|c|c|c|c|c|c}
\hline \hline \multirow{2}{*}{ mBasis } & \multicolumn{4}{|c|}{ Dimensionless size of support domain $\left(\alpha_{\mathrm{s}}\right)$} & \multirow{2}{*}{ Exact } \\
\cline { 2 - 6 } & 2.5 & 3.0 & 3.5 & 4.0 & 4.5 & \\
\hline 3 & 0.487777 & 0.498836 & 0.510496 & 0.497278 & 0.535784 & 0.4789 \\
6 & 0.488044 & 0.470402 & 0.470146 & 0.502782 & 0.509977 & \\
10 & 0.002825 & 0.454212 & 0.568492 & 0.495713 & 0.498427 &
\end{tabular}

\section{Conclusion}

In this study, the selectable parameters of EFG method were investigated at different values for the solution of bending problems of the Reissner-Mindlin plate. It is shown that the number of Gauss points, type of weight function, and the use of regular and irregular node distribution does not show any significant accuracy loss for the displacement results. But, several variations are shown for the moment results. From the results the values for the number of monomials (mBasis), the dimensionless size of support domain $\left(\alpha_{\mathrm{s}}\right)$, the number of Gauss points (nGauss), the value of penalty coefficient $\left(\alpha_{p}\right)$ can be suggested as $3,3.0,5 \times 5,1.0 \times 10^{6}$, respectively. Also, it is shown that the quartic spline weight function is smoother than the cubic spline weight function. These values may not be the optimum values for every situation, however, in general, give results with sufficient accuracy.

\section{References}

[1] T. Belytschko, Y.Y. Lu, L. Gu, Int. J. Num. Meth. Eng. 37, 229 (1994).

[2] G.R. Liu, Mesh Free Methods, Moving Beyond the Finite Element Method, CRC Press, New York 2002.

[3] H. Nguyen-Xuan, T. Rabczuk, S. Bordas, J.F. Debognie, Comput. Meth. Appl. Mech. Eng. 197, 1184 (2008). 